\title{
A Revised Model of Photon Resulted by an Etherono-Quantonic Theory of Fields
}

\author{
Arghirescu Marius \\ State Office for Inventions and Trademarks, OSIM, Bucharest, Romania \\ Email: arghirescu.marius@osim.ro
}

Received 2 October 2015; accepted 18 October 2015; published 23 October 2015

Copyright (C) 2015 by author and OALib.

This work is licensed under the Creative Commons Attribution International License (CC BY). http://creativecommons.org/licenses/by/4.0/

(c) $\underset{\mathrm{EY}}{\text { (i) }}$ Open Access

\section{Abstract}

By an etherono-quantonic theory of the author [1], which deduces that the magnetic field of a magnetic moment is generated by an etherono-quantonic vortex $\Gamma_{M}=\Gamma_{A}+\Gamma_{B}$ of etherons and of quantons with mass $m_{h}=h / c^{2}$, giving the magnetic induction $B$, a revised model of pseudoscalar photon derived from the Munera's model and the Hunter-Wadlinger model is proposed, formed by two vectorial photons with diameter $\lambda / \pi$, deformed to the speed direction to the value: $\lambda / 2$ proportional with the speed. The revised model of photon is compatible with the ondulatory and the corpuscular properties of the particles and allows a phenomenological microphysical explanation for the Kerr and the Cotton-Mouton effects and other similar effects. It is explained also microphysically the magnetic rotating polarization (the Faraday effect). The model indicates the variation of the quantum vacuum refraction index in the presence of a strong electric $E$-field or magnetic $B$-field.

\section{Keywords}

Pseudo-Scalar Photon, Etherono-Quantonic Vortex, Magnetic Field, Kerr Effect, Cotton-Mouton Effect, Munera Model

Subject Areas: Theoretical Physics

\section{Introduction}

The dualism: wave-corpuscle of the light determined the proposal of some pseudo-scalar photon models (for a photon spin: $S=h / 2 \pi$ ) of solitonic type.

To explain the wave-particle dualism of the pseudo-scalar photon, H. A. Mùnera considers the photon as a semi-classic vortexial doublet of particle-antiparticle pair, which explains the frequency and the repose mass of a photon, [1], considering preonic vortexes of quasi-constant density, formed in the quantum vacuum. A photon model was proposed also by Lenhert, [2] which deduced a photon diameter: $d=\lambda_{0} / \pi$ and which indicated a 
non-null rest-mass of the photon, resulted by the fact that it was associated with the photon spin and it explained the photoelectric effect and the photon's splitting.

Geoffrey Hunter and L. P. Wadlinger [3] proposed a solitonic model of photon corresponding to the Einstein's concept of photon considered as a localized and confined electromagnetic wave in a circular volume of an ellipsoid with the length along the propagation axe-equal to the associated wave-length, $\lambda$, and the photon diameter: $d_{f}=$ $\lambda_{0} / \pi$.

According to an etherono-quantonic theory of the author based on the galileian relativity, which sustains the possibility of particles cold genesis (CGT, [4]), the magnetic field is generated by an etherono-quantonic vortex $\Gamma_{M}=\Gamma_{A}+\Gamma_{B}$ of $s$-etherons (sinergons-with mass $m_{s} \approx 10^{-60} \mathrm{~kg}$ ) giving the magnetic potential $A$ by an impulse density: $p_{s}(r)=\left(\rho_{s} \cdot c\right)_{r}$ and of quantons (h-quanta with mass: $m_{h}=h / c^{2} \approx 7.37 \times 10^{-51} \mathrm{~kg}$ ), giving the magnetic induction $B$ by an impulse density: $p_{c}(r)=\left(\rho_{c} v_{c}\right)_{r}$, generated by a magnetic moment of an atomic particle but also by a magnet or an electromagnet.

The known relation: $B=\operatorname{rot} \cdot A$ is explained in the theory by the conclusion that the $\xi_{B}$-field lines of the magnetic induction $B$ having a quantum density $\rho_{B}(r)$ are materialized as quantonic vortex-tubes formed around some oriented pseudostationary quanta of the electric field (named "vectons", i.e. vectorial photons, with $m_{v}=3$ $\times 10^{10} m_{h}=2.2 \times 10^{-40} \mathrm{~kg}$ ) accumulated from the quantum vacuum by the quantonic vortex $\Gamma_{B}$ of the $B$-field, generated by the gradient of the sinergonic impulse density of the $\Gamma_{A}$-vortex: $p_{s}=\rho_{S}(r) \cdot c$, according to the equations:

$$
\begin{gathered}
A_{j}=\frac{1}{2} B_{k} \cdot r=\frac{1}{2} k_{1} \rho_{B}(r) \cdot c \cdot r=\frac{1}{2} k_{1} \rho_{c}(r) \cdot v_{c}(r) \cdot r=\frac{1}{2} k_{1} \rho_{s}(r) \cdot r_{\mu} \cdot c \\
B_{k}=r o t \cdot A=\frac{1}{2} k_{1} r_{\mu} \cdot \partial_{i}\left(\rho_{s}(r) \cdot c\right)_{j} ; \quad\left(\partial_{i}=\partial / \partial x_{i} ; x_{i} / / r\right)
\end{gathered}
$$

with: $k_{1}=S^{0} / e=1.57 \times 10^{-10}\left[\mathrm{~m}^{2} / \mathrm{C}\right]_{\mathrm{si}},\left(S^{0}=4 \pi a^{2} ; a=1.41 \mathrm{fm}\right) ; r_{\mu}$-the Compton radius of the considered electric charge and $v_{c}(r) \approx\left(r_{\mu} / r\right) \cdot c$, (i.e. with $\left.\rho_{c}(r) \approx \rho_{s}(r)\right)$.

Also, the expression of the electric field results in the theory by equation:

$$
E_{c}=k_{1} \rho_{c}(r) \cdot v_{c}^{2}=k_{1} \rho_{a}^{0} \frac{a^{2}}{r^{2}} \cdot v_{c}^{2} ; \quad \rho_{a}^{0}=\rho_{c}(a) ; \quad v_{c}=c ; \quad k_{1}=\frac{4 \pi a^{2}}{e} ; \quad \rho_{c}(r)=\rho_{a}^{0} \frac{a^{2}}{r^{2}}
$$

with: $\rho_{a}^{0}=\mu_{0} / k_{1}^{2}$.

The gauge constant $k_{1}$ results in the theory from a Q(M)-charge model given by a spheric-symmetric distribution of charge's quanta: $n \cdot m_{c}\left(r_{c}\right)$ around the particle having the electron radius: $r_{a}=a$, i.e.: $\rho_{a} \cdot r^{2}=\rho_{a}^{0} \cdot a^{2}$, which generates a pressure force over the semi-surface $S^{x}=S^{0} / 2=2 \pi r_{0}^{2}$ of a pseudocharge $q_{s}\left(m_{0}\right)$ approximated as equal with its scattering cross section: $\sigma=\pi\left(r_{0}+r_{c}\right)^{2} \approx S^{x}$, given by the impulse density variation: $\Delta p_{c}=p_{c}(r)-p_{c}(-r)=2 n \cdot m_{c} v_{c}$ :

$$
F(r)=S^{x} \frac{\Delta\left(p_{c}\right)}{\Delta t}=S^{x} \frac{\Delta\left(n m_{c} \cdot v_{c}\right)_{r}}{\Delta t}=S^{0} \rho_{v}(r) \cdot v_{c}^{2}=q_{S} \cdot E(r) ; \quad n \cdot m_{c}=n_{0} \Delta r \cdot m_{c}=\rho_{v} \Delta r
$$

the expression of $k_{1}$ resulting by the equation of equality between the electrostatic energy and the vectorial photons kinetic energy at the electron's surface, i.e.:

$$
E_{E}(a)=E_{k}(a) \Rightarrow \frac{1}{2} \varepsilon_{0} E^{2}(a)=\frac{1}{2} \rho^{0}(a) \cdot c^{2}=\frac{1}{2} E(a) / k_{1} \Rightarrow k_{1}=4 \pi a^{2} / e .
$$

The Lorentz force results according to the theory, in the form:

$$
F_{L}=2 a \cdot \Gamma_{a}^{*} \cdot \rho_{B} \cdot v_{e}=q \cdot B \cdot v_{e}=e \cdot k_{1}\left(\rho_{e} v_{v}^{r}\right)_{r} \cdot v_{e} ; \quad \Gamma_{a}^{*}=\varsigma \cdot 2 \pi \cdot a \cdot c ; \quad \rho_{B}=\rho_{e}(r) \cdot\left[v_{v}^{r} / c\right] ; \quad \varsigma= \pm 1
$$

as force of Magnus type over an $e$-charge with the surface circulation $\Gamma_{a}^{*} ;\left(v_{v}^{r}\right.$-the relative speed of quantons forming the $\Gamma_{B}$-vortex in report with the vectons of the $E$-field; $\rho_{B}$-the density of $\xi_{B}$-vortex-tubes considered with the vorticity speed: $\omega r=c$ ).

Also, the theory deduces the variation of the Compton radius, $r_{\lambda}$ and of the fermion's magnetic moment $\mu=$ 
$e \cdot c \cdot r_{\lambda} / 2$, inverse proportional with the density in which is placed the particle's superdense kernel, (the particle's centroid): $\left(r_{\lambda} ; \mu\right) \sim \rho_{c}^{-1}$ as explanation of the dependence: $\mu_{p} \sim\left(m_{p}\right)^{-1}$.

The theory retrieves the Munera's conclusion regarding a composed model of pseudoscalar photon formed as doublet of vectorial photons with spinorial mass (named "vexons" in the theory) and sustains also the possibility of a cold genesis of particles, which results theoretically in a chiral soliton model as Bose-Einstein condensate of photons in the electron's case and of "gammons" (pairs of degenerate electrons, i.e. of quasielectrons: $\gamma_{c}=\left(e^{+}-\right.$ $\left.e^{-}\right)$) in the nucleon's case.

\section{A Revised Model of Photon}

It may be observed that if the Múnera's model of photons is dimensioned like in the Hunter-Wadlinger model, considering the simple photon as a doublet of two vectorial photons with mutually anti-parallel spins $S=\hbar / 2$ and a mean diameter: $d_{w}=\lambda / \pi$ and considering the hard-gamma quanta as a doublet: negatron-positron, $\gamma_{c}=\left(e^{+}-e^{-}\right)$, with opposed spins and the energy: $\varepsilon_{\gamma}=h v=2 m_{e} c^{2}$, it results that the electron of a $\gamma_{c}$-doublet may be assimilated with a vectorial (semi) photon, $m_{w}^{e}$, with a $r_{\lambda}$-radius which results $1 / 2$ from the Compton radius of a free electron: $r_{\lambda}=\lambda_{v} / 2 \pi,\left(\lambda_{v}=2 \lambda\right)$, i.e. a degenerate electron Compton radius, $r_{\lambda}^{\prime}$, possibility conform to the dependence $r_{\lambda} \sim \rho_{c}^{-1}$, specific to CGT.

Because that according to CGT, the magnetic field B is given by a vortex of sinergons and of quantons which has the rotation speed: $\omega r=c$ inside the pseudo-cylindical volume of $r_{\lambda}: v_{\lambda} \approx 2 \pi r_{\lambda}^{3}=\lambda^{3} / 4 \pi^{2}$, from the equality between $w_{\mu}^{f}=(1 / 2) \mu_{0} H^{2} \sim(1 / 2) m_{S}\left(\omega_{h} \cdot r\right)^{2}$ and $w_{E}^{f}=(1 / 2) \varepsilon_{0} E^{2} \sim(1 / 2) m_{S} c^{2}$ given by the translation energy of a spinorial $\Gamma_{S}$ vortex of quantons, which do not contribute to the inertial mass $m_{v(w)}$ of the photon core, it results for the proposed revised model of photon, the equation:

$$
E_{E}^{c}=E_{\mu}^{c} \Rightarrow \frac{\Sigma m_{h} c^{2}}{2}=\frac{m_{s}^{\prime}(\omega \cdot r)^{2}}{2}=\frac{h v}{4}=\frac{m_{w} c^{2}}{2} ; \quad \omega \cdot r=c .
$$

In this case, it results that: $E_{v}=2\left(E_{E}+E_{\mu}\right)=2 m_{s} c^{2}=h v=2 m_{w} c^{2}$, by the conclusion that the spinorial (vortexial) $m_{s}$-mass of the vexon, given as vortex of quantons and quantonic clusters, is equal with its inertial mass: $m_{w}$-which explains the gravitational force generated over photons and the atomic electron transition to an superior energetic level by photons, considered in CGT as cluster of vectons with super-dense kernel.

This equality: $m_{s}=m_{w}$ is argued in CGT by the fact that for a vectorial photon with the $\rho_{w}$-density variation with $r^{-1}$-for a pseudo-cylindrical $v_{\lambda}$-volume or with $r^{-2}$-for a spherical $v_{\lambda}$-volume, the vectorial photon spin results of value:

$$
S_{v}^{*}=\int_{r_{a}}^{r_{v}} r \cdot c \cdot \mathrm{d} m \cong 4 \pi r_{a}^{2} \rho\left(r_{a}\right) \cdot c \cdot \frac{r_{v}^{2}}{2} \cong m_{s} \cdot c \cdot \frac{r_{v}}{2}=m_{s} c \cdot \frac{\lambda_{v}}{4 \pi}=m_{s} \cdot c \cdot \frac{h}{4 \pi \cdot m_{w} c}=\frac{1}{2} \frac{h}{2 \pi}
$$

so-equal with the value considered for fermions by the quantum mechanics.

The explaining of this equality: $m_{s}=m_{w}$, may be given by the chiral sub-solitons forming condition [5] which specifies that the energy $E_{\Gamma}=m_{\Gamma} c^{2}=2 E_{\mu}$ of the mass-generating chiral soliton field, given in this case by the sinergono-quantonic vortex $\Gamma_{\mu}=\Gamma_{A}+\Gamma_{H}=2 \pi r \cdot c$, should be double, at least, comparing to the mass energy: $E_{w}$ $=m_{w} c^{2}$ of the generated sub-solitons, i.e.: $E_{\Gamma}=2 E_{w}$.

However, the explaining of the wave-particle dualism of the photon in only partially solved in this stage, because that the photon length must be: $l_{v}=\lambda$, so-greather than $2 d_{w}=2 \lambda / \pi$.

The problem may be solved by the conclusion that the vectorial photon volume $v_{\lambda}$ is a little expanded over the value $d_{w}$ to at least one direction. We have in this sense three theoretical possibilities:

a) the collinear orientation of the vectorial photons; this case correspond to the Hunter-Wadlinger photon model, but it gives a longer vectorial photon, longitudinally polarised, which not explain causally the ondulatory properties of the photon, given by: $H \perp c$;

b) the vexon volume expansion along axis $x, y \perp c$; this possibility suppose that the vortexial energy $E_{\mu}$ of vectorial photon is contained by a volume: $v_{\lambda}^{*} \approx \pi(\lambda / \pi)(\lambda / 4)^{2} \approx \lambda^{3} / 16$ and corresponds to a degenerate Compton radius of the vectorial photons, of value: $r_{\lambda}^{\prime} \approx \lambda / 4<r_{\lambda}=\lambda_{v} / 2 \pi$. But experimental determinations (1985-1986, [6]) confirmed the value: $\lambda / \pi$ for the diameter of the photon's section, (with $\lambda=1 / 2 \lambda_{v}$ ); 
c) the vexon volume expansion along the axe $x / / v_{p} \leq c$; this possibility suppose that the vortexial energy $E_{\mu}$ of vectorial photon is contained by an ellipsoidic volume with the length $: l_{w}=\lambda / 2$, i.e.:

$v_{\lambda}^{e} \approx \pi(\lambda / \pi)(\lambda / 2 \pi)(\lambda / 4) \approx \lambda^{3} / 8 \pi$, the inertial $m_{w}$-mass of vexon being closer to the focal point of the ellipsoid which is the first in contact with the pseudo-stationary (brownian) etherons and quantons of the quantum vacuum, of total density: $\rho_{r}=\rho_{r c}+\rho_{r s}$, which acts as a relativist wind deforming the vexonic quantum volume along $x / / v_{p}$, by the sinergonic component: $P_{s}=\rho_{r s} v_{p}^{2}$, until a value: $l_{w}=\lambda / 2$, (Figure 1), i.e.:

$$
l_{w}=\left(2 r_{\lambda}+\Delta l_{w}\right) \propto P_{s}=\rho_{r s} v_{p}^{2}=\left(k_{v} / v_{k}\right) \cdot v_{p}
$$

relation which results in the hypothesis of a relativist etherono-quantonic impulse density: $p_{r}=\rho_{r} v_{p}$ given by the equation:

$$
p_{r}=\rho_{r} v_{p}=\left(k_{v} / v_{k}\right) ; \quad\left(\rho_{r}=\rho_{r c}+\rho_{r s}\right)
$$

in which: $k_{v}$-proportionality constant; $v_{k}$-the kinematic viscosity of quantum vacuum. The considered ansatz (10) will be justified afterward.

By Equations (9), (10), the revised model of photon may explain also the photon wavelength variation with the photon's speed in an optical medium with a specific index of refraction, $n$, i.e.:

$$
v_{V}=c / n \Rightarrow \lambda=\lambda_{0} / n ; \quad\left(v=2 m_{V} / m_{h}=\text { constant; } v_{V}=-v_{p}\right)
$$

in the form:

$$
\Delta v=\left(c-v_{v}\right) \propto\left(\rho_{r}-\rho_{r}^{0}\right) ; \quad l_{w} / l_{w c}=v_{v} / c ; \quad\left(l_{w c}=\lambda_{0}(c) ; l_{w}=\lambda\left(v_{p}\right) ; v_{v}=-v_{p}\right)
$$

in which $\rho_{r}^{0}$ is the initial density of the etherono-quantonic medium in the quantum vacuum and $v_{v}$ is the photon's speed in the optical medium.

For: $l_{w} / 2 \approx \lambda_{0} / \pi,\left(v_{v} \leq 0.64 c\right)$, the dynamic equilibrium for the vortexed quantons or clusters of quantons inside the Compton radius: $r_{\lambda}=\lambda_{0} / 2 \pi$, is given by a magneto-gravitic force of Magnus type generated by the sinergonic vortex over the quantons rotated with $v=c$ to the vortex line: $l_{r}=2 \pi r$, in the form:

$$
F_{S l}=2 r_{c} \cdot \Gamma_{c}\left(r_{c}\right) \cdot \rho_{s}(r) \cdot c=4 \pi \cdot r_{c}^{2} \cdot c^{2} \cdot \rho_{s}^{0} \cdot\left(r_{w} / r\right)=m_{h} c^{2} / r ; \quad r \leq r_{\lambda}
$$

by the resulted condition: $4 \pi r_{c}^{2} \cdot \rho_{s}^{0} \cdot r_{w}=m_{h}=h / c^{2}$, with: $\Gamma_{c}\left(r_{c}\right)=2 \pi r_{c} \cdot c ; r_{c}$-the quanton radius; $\rho_{s}^{0}$-the density of sinergons at the vexon's inertial mass surface of radius: $r_{w} \leq a$; $m_{h}-$ the quanton mass; $\Gamma_{c}\left(r_{c}\right)$-the circulation of sinergons at the quanton's surface.

Also, because that in the case of light propagation in an optical medium in the presence of an intense electric $E$-field or magnetic $B$-field, the etherono-quantonic density $\rho_{\mathrm{r}}$ of the quantum vacuum is supplemented with a value: $\Delta \rho_{r} \sim \varepsilon E^{2}$ or $\Delta \rho_{r}^{\prime} \sim \mu H^{2}$ according to CGT, it is possible to explain by the model also the Kerr effect [7] and the Cotton-Mouton effect [8], of the refractive index changement in an applied $E$-field or $H$-field or by the intensity I of the light beam, (the AC Kerr effect), by the hypothesis that the photon speed: $v_{p} \leq c$ results by a quantum Stokes force: $F_{S}=K_{S} \cdot \eta d \cdot v_{p},\left(\eta=\rho_{r} v_{k}\right.$-the dynamic viscosity; $v_{k}$-the kinematic viscosity of quantum vaquum; $\rho_{r}$-the etherono-quantonic density), acting over the $m_{p}$ inertial mass of photon and generating a quasiconstant mean deceleration.

We observe that the solution c), conformed with the Figure 1, by the resulted dependence:

$l_{w}=\left(2 r_{\lambda}+\Delta l_{w}\right) \sim v_{p}$, determined by the relativist etheronic impulse density: $p_{r}=\rho_{r} v_{p}$, explains also the photon wavelength variation with the speed of photon in a given optical medium with a specific index of refraction, $n$ : $v_{v}=c / n \Rightarrow \lambda=\lambda_{0} / n ;\left(v=2 m_{v} / m_{h}=\right.$ constant $)$, in the form (12).

Also, by the model, it results that a pair: photon-photon or photon-antiphoton, formed as two identical photons weakly coupled in phase or antiphase, may be splitted into two photons which thereafter may interfere, in accordance with the double slit experiment result, (the vexonic inertial mass of each photon cross the slit carrying its electromagnetic vortex).

\section{The Kerr and Cotton-Mouton Effects Explaining}

Because that in the case of light propagation in an optical medium in the presence of an intense electric $E$-field 


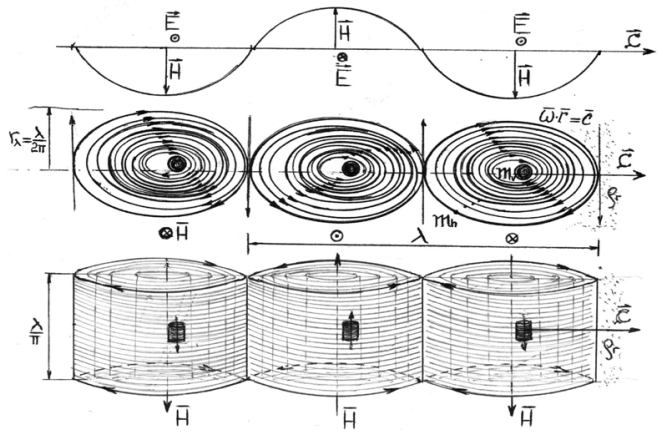

Figure 1. Revised model of multi-photon, $\left(l_{p}=1.5 \lambda\right)$.

of magnetic $B$-field, the etherono-quantonic density $\rho_{r}$ of the quantum vacuum is supplemented with a value: $\Delta \rho_{r}$ $\sim \varepsilon E^{2}$ or $\Delta \rho_{r}^{\prime} \sim \mu H^{2}$ according to CGT, it is possible to explain by the previous model also the Kerr effect [7] and the Cotton-Mouton effect [8], of the referactive index changement in an applied $E$-field or $H$-field or by the intensity $I$ of the light beam, (the AC Kerr effect), by the hypothesis that the photon speed: $v_{v} \leq c$ corresponds to a quantum Stokes force:

$$
F_{S}=K_{S} \cdot \eta \cdot d \cdot v_{v}
$$

( $\eta=\rho_{r} v_{k} ; v_{k}$-kinematic viscosity of quantum vaquum), acting over the $m_{p}$ inertial mass of photon and giving a deceleration of mean value $a_{e}$, in the form:

$$
a_{e}=\frac{F_{S}}{m_{p}}=\frac{K_{S} \rho_{r} v_{k} d \cdot v_{v}}{h} \cdot c \lambda_{0} \approx K_{n} .
$$

The refraction index results from Equation (15) in the form:

$$
n=\frac{c}{v_{V}}=\frac{K_{S} \rho_{r} v_{k} d \cdot}{h \cdot K_{n}} \cdot c^{2} \lambda_{0}=\sqrt{\varepsilon_{r} \mu_{r}} .
$$

We observe that if $K_{n}$ quasi-constant, because that in CGT is considered a radius: $r_{w} \approx k_{r} a=(0.4-1) \cdot a$ with $a$ $=1.41 \mathrm{fm}$, for the inertial mass of the photons, $\left(k_{r}=0.41\right.$ for vectons-equation (3) and $k_{r} \approx 0.7$ for light photons), it results that:

$$
\eta=\rho_{r} v_{k}=\frac{h \cdot K_{n}}{K_{S} d \cdot c^{2} \lambda_{0}} \sqrt{\varepsilon_{r} \mu_{r}} \approx \frac{K_{p}}{\lambda_{0}} n ; d=2 k_{r} a ; \rho_{r} v_{v}=\frac{K_{p}}{\lambda_{0}} \frac{c}{v_{k}}=\frac{k_{V}}{v_{k}} .
$$

So, by the prevous theoretical assumption, it is justified the ansatz (10) looking the dependence: $l_{w}\left(v_{v}\right) \sim v_{v}$. Also, for $v_{v}$ it results that:

$$
v_{v} \approx \frac{K_{p}}{v_{k}} \cdot \frac{c}{\rho_{r} \lambda_{0}} ; \quad n_{1}=\frac{c}{v_{1}}=\frac{v_{k} \rho_{r 1} \lambda_{0}}{K_{p}} ; \quad n_{2}=\frac{c}{v_{2}}=\frac{v_{k} \cdot \rho_{r 2} \lambda_{0}}{K_{p}}=\frac{v_{k} \cdot\left(\rho_{r 1}+\Delta \rho_{r}\right) \lambda_{0}}{K_{p}}=n_{1}+\Delta n .
$$

If: $\Delta \rho_{r} \approx k_{E} \cdot \varepsilon E^{2} / c^{2}$ and: $\Delta \rho_{r}^{\prime} \approx k_{H} \cdot \mu H^{2} / c^{2},\left(k_{E} ; k_{H} \leq 1\right.$ proportionality constant, depending on the optical medium), it results by Equation (18) that:

$$
\begin{aligned}
& \Delta n_{E} \approx \frac{k_{E} v_{k} \varepsilon \cdot E_{0}^{2}}{c^{2} \cdot K_{p}} \lambda_{0}=\lambda_{0} K_{E} E_{0}^{2} ; \quad\left(K_{E}=\frac{k_{E} v_{k} \varepsilon}{c^{2} \cdot K_{p}}\right) ; \\
& \Delta n_{H} \approx \frac{k_{H} \mu v_{k} H_{0}^{2}}{c^{2} \cdot K_{p}} \lambda_{0}=\lambda_{0} K_{H} H_{0}^{2} ; \quad\left(K_{H}=\frac{k_{H} v_{k} \mu_{0}}{c^{2} \cdot K_{p}}\right)
\end{aligned}
$$

where $K_{E}$ and $K_{H}$ are the Kerr and the Cotton-Mouton constants of the optical medium.

We obtain in this case, a phenomenological microphysical explanation for the Kerr and the Cotton-Mouton 
effects, by a solitonic model of photon, compatible with the ondulatory and the corpuscular properties of the particles. The decomposing of a monochomatic polarised light beam in two waves: ordinary and extraordinary waves in a strong magnetic field, may be also explained by the revised model of photon, considering also the difference of the magnetic interaction with the external $B$-field of the two vectorial photons which gives the light photon.

Also, in the inverse Cotton-Mouton effect, when an oscillating field passes through a dielectric medium in such a way that it's oscillations are similar to the polarization induced by the C-M effect, then a static magnetic field is created perpendicular to the direction of propagation of light. This effect is also explained by the revised model of photon, by the fact that the $\Gamma \mu$-vortexes of light quantum vexons induces parallely oriented vortexes of a $H$-field in the quantum medium of the dielectric body.

It results also-from the model, that the photon may be considered un-deformed until a speed:

$$
v_{p}^{c}=2 d_{p}\left(c / \lambda_{0}\right)=2 \lambda_{0} \cdot c / \pi \lambda_{0}=(2 / \pi) c \approx 0.64 c .
$$

The refraction angle may be also explained by a model of double multiphoton (two multiphoton rows coupled in phase) which may explain also the photon's splitting at its passing through a double slit. that:

If the dielectric body has a speed $v_{D} / / v_{p}^{\prime}$ with $v_{p}^{\prime} \neq v_{p}$, if $K_{n}^{\prime}\left(v_{p}^{\prime}\right) \approx K_{n}\left(v_{p}\right)$, it results by Equation (15)

$$
v_{p}^{r}=v_{D}+v_{p}^{\prime}=v_{p}
$$

(i.e. the invariance of $v_{p}^{r}$-speed of light).

The possibility: $K_{n}^{\prime}\left(v_{p}^{\prime}\right) \approx K_{n}\left(v_{p}\right)$ corresponds to the conclusion that the etheronic pressure $P_{e}$ is giveninside the dielectric body, by atomic vortexes, phenomenon which may explain the fact that - when the photon get out from the optical body, it regains its normal speed, $c=1 / \sqrt{\left(\varepsilon_{0} \mu_{0}\right)}$.

Also, the possibility to "freeze" light inside a crystal [9] may be explained by the revised vortexial model of photon, by the hypothesis of ring multi-photons forming in the magnetic field of the electrons or/and of the atoms.

We observe also that considering a speed: $v_{v}=c / n$ also for the vectorial photons in an optical body, the Equation (4) becomes:

$$
\begin{gathered}
E_{E}(a)=E_{k}(a) \Rightarrow \frac{1}{2} \varepsilon \cdot E^{2}(a)=\frac{1}{2} \rho^{0}(a) \cdot v_{v}^{2}=\frac{1}{2} E(a) / k_{1} ; \quad k_{1}=4 \pi a^{2} / e \\
\varepsilon \cdot E(a)=\varepsilon_{0} \varepsilon_{r} \cdot k_{1} \rho^{0}(a) \cdot v_{v}^{2}=\varepsilon_{0} \varepsilon_{r} k_{1}\left(\mu_{0} / k_{1}^{2}\right) \cdot v_{v}^{2}=1 / k_{1} \Rightarrow \varepsilon_{r}=\left(v_{v} / c\right)^{-2}=n^{2}
\end{gathered}
$$

i.e. is re-obtained a known relation, verified for very low frequencies of the photons, i.e. for the far-IR domain; (for higher frequencies: $\varepsilon_{r}=\varepsilon_{r}(v)$ ).

Also, if $\rho^{0}(a)=\left(\mu / k_{1}^{2}\right)=\left(\mu_{0} \mu_{r} / k_{1}^{2}\right)$, we obtain by Equations (21a), (21b) that: $n^{2}=\varepsilon_{r} \mu_{r}$.

The first case $(n=\sqrt{\varepsilon})$ may be interpreted by Equation (21) in the sense that the low frequency photons not modify the local values of $\rho^{0}(a)$ and $E(a)$ when they pass through the $E$-field of the electron or of the proton surface.

The fact that the Kerr and Cotton-Mouton effect generates a bi-refringence of the optical crystal, the fact that the Kerr effect is established with a delay of few seconds in solids and polar viscous liquids and the fact that it is known also a mechanical or a dynamic birefringence or generated by quenching, indicates that the bi-refringence is generated by the atomo-molecular arrangement (orientation) which generates an anisotropy of the mean quantum $\bar{\rho}(r)$ density distribution, according to Equation (18). This conclusion is in concordance also with the existence of substances as quartz which generates rotating polarization effect depending on the local crystal's density and its thickness: $\alpha \sim \rho \cdot l$, with $\rho=\rho(\lambda)$ with $k=\alpha / \rho \cdot l$ not depending of the physical state of the substance.

According to the revised model of photon, the photon's polarization plane is formed by the spins and the magnetic moments of the coupled vectorial photons which gives the pseudoscalar photon.

The scalar quantum corresponds to a couple of two pseudoscalar photons coupled in antiphase. In this case, the electromagnetic effect of $\Gamma_{\mu}$-vortexes of vexons is reciprocally canceled but the scalar quantum has an im- 
pulse: $m_{s} c=h v / c=4 m_{w} c$ given by the inertial mass of the component vexons.

It is argued in CGT that a scalar quantum may be emitted by charges vibrated by energetic shocks.

In relation to these previous conclusions, the general microphysical way in which was deduced the Equation (19) indicates the possibility to modify the refraction index of the quantum vacuum (normally: $n_{0}=1$ ) by the presence of a local intense electric $E$-field or magnetic $B$-field, according to the model. We observe also that the dependences: $n \sim \rho_{r}$ and $\left(r_{\lambda} ; \mu\right) \sim \rho_{c}^{-1}$ may explain the relation: $l_{w} \sim v_{p}$ also by the explicative variant b), if we admit a variable radius: $r_{\lambda} \sim v_{p}$ of the photon's section.

Also, the magnetic rotating polarization (the Faraday effect) (Figure 2), consisting in the rotation of the polarization plane of a light beam in a magnetic field parallel with its speed: $H / / c$, with an angle:

$$
\alpha=\rho_{v} \cdot l \cdot H
$$

( $\rho_{v}=\rho_{v}(\lambda)$-the Verdet constant, the rotation sense depending on the $H$-field sense), may be explained by the proposed revised model of photon in connection with the quantum vortexial nature of the magnetic field considered in the etherono-quantonic theory [4], by the conclusion that the etherono-quantonic dynamic pressure: $P_{d}$ $=1 / 2 \rho_{B} C^{2}$ of the $\xi_{B}$ vortex-tubes of the magnetic induction $B=\operatorname{rot} \cdot A$, which generates the magnetic field lines, action over the inertial $m_{w}$ and the spinorial $m_{S} \approx m_{w}$ mass of the vectorial photons (which gives the pseudoscalar photon: $h v=m_{p} c^{2}$ ) by a pseudo-electric field $E_{q}$ tangent to the $\xi_{B}$ vortex-tube, forming the $B$-field lines, i.e.:

$$
E_{q i}=2 k_{1} \cdot P_{d i}=B_{k} \cdot c ; \quad B_{k}=k_{1} \cdot \rho_{B} \cdot c ; \quad \omega=(\alpha / t) \propto E_{q} \propto B
$$

according to Equations (1)-(3). So, $E_{q}$ generates a rotation of the photon's polarization plane with $\omega$ depending on the sense of the $\xi_{B}$ vortex-tubes and on their density: $\rho_{B} \sim B$, with an angle:

$$
\alpha=\omega \cdot t=k_{F} \cdot E_{q} \cdot t=k_{F} \cdot(\mu \cdot H) \cdot c \cdot\left(l / v_{p}\right)=\rho_{V} \cdot l \cdot H ; \quad\left(\rho_{V}=k_{F} \cdot \mu \cdot n\right) .
$$

Similarly may be explained also the Einstein-de Haas effect, determining the rotation of a ferromagnetic bar introduced in a magnetic $B$-field, [4].

\section{The Gamma Quanta}

In the case of gamma quanta, the phenomenon of pairs conversion: $\gamma \rightarrow e^{+}+e^{-}$, indicates the existence of two types of gamma quanta: soft gamma quanta, with normal ondulatory (electromagnetic) properties, which are composed according to the revised model of photon (Figure 1) and hard-gamma quanta, with altered electromagnetic properties, i.e. acting mainly by the energy of the inertial mass and composed from a partially degenerate pair: negatron-positron with the inertial mass in a volume with radius equal with the electron's Compton radius, $r_{e}=\lambda_{e} / 2 \pi=\hbar / m_{e} c$, which is also the vibration amplitude $A_{v}^{0}$ of the system necessary for fermions separation. The argument for this conclusion is the fact that the conversion $\gamma \rightarrow e^{+}+e^{-}$may occur in the electric field $E$ of a nucleus, so the fermions which composes the hard-gamma quanta has also electric charge.

At very low temperature, $T \rightarrow 0 \mathrm{~K}$, when $A_{v} \ll A_{v}^{0},\left(A_{v} \rightarrow a\right)$, because the physical contact between component electrons surface, their inertial masses and magnetic moments may be diminished because the decreasing of the density variation mean radius, $\eta^{*}$, according to the relation:

$$
\rho(r)=\rho^{0}\left|\Psi_{e}\right|^{2} ; \quad\left|\Psi_{e}\right|^{2}=k_{d} \cdot \Psi_{e}^{-} \cdot \Psi_{e}^{+} ; \quad\left(\Psi_{e}^{-}=R \cdot e^{-i S / \hbar} ; \quad \Psi_{e}^{+}=R \cdot e^{i S / \hbar}\right)
$$

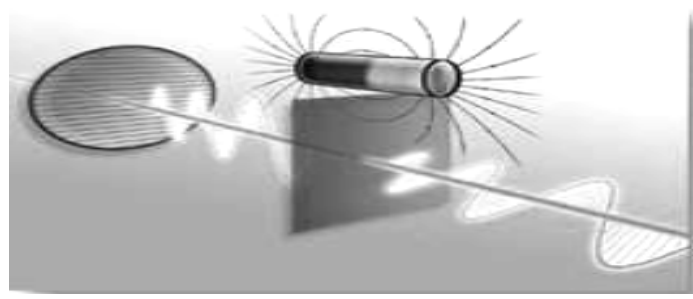

Figure 2. The Faraday effect (the vorticity of the $\xi_{B}$ field line determines the rotation of the photon's polarization). 
where: $\Psi_{e}^{-} ; \Psi_{e}^{+}$-the wave function of the free negatron and positron structure, $\Psi_{e}^{*}$-the wave function of the degenerate electron and $k_{d}$-degeneration coefficient, which depends on the distance $l$ between the component electrons, for a system with more electrons, $k_{d}$ depending also of the number of system components, (for example in the case of a meson or a baryon formed as Bose-Einstein condensate of gammons, [4]). The wave function of the bound electron becomes in this case:

$$
\Psi_{e}^{ \pm *}=R^{*} \cdot e^{ \pm \frac{S}{\hbar}} ; \quad \text { with }:\left(R^{*}\right)^{2}=e^{-\frac{r}{\eta^{*}}}=k_{d} \cdot R^{2} ; R^{2}=e^{-\frac{r}{\eta}} ; \eta^{*}<\eta .
$$

It is possible to estimate empirically the expression of $k_{d}$ by CGT [4], with the case of a proton formed as B-E condensate of $n=1052$ gammons, (degenerate hard-gamma quanta), the mean radius $\eta$ of the electron decreasing from $\eta_{e}=0.965 \mathrm{fm}$ (of the free electron) to $\eta_{n}=0.849 \mathrm{fm}$ for the quasielectron of the protonic B-E condensate, according to CGT, value which is very close to the experimentally determined root-mean-square radius of proton's charge density variation: $\eta_{n}^{e}=0.841 \mathrm{fm}$ [10] and which results for a proton with a considered effective radius $: r_{p} \approx a=1.41 \mathrm{fm}$, by the mass integral equation:

$$
m_{p}=\int_{0}^{a} 4 \pi r^{2} \rho_{p}(r) \cdot \mathrm{d} r=1.67 \times 10^{-27} \mathrm{~kg} ; \rho_{p}(r)=\rho_{p}^{o} \cdot e^{-\frac{r}{\eta_{p}}}=\rho_{p}^{o} \cdot\left|\Psi_{p}\right|^{2} ; \rho_{p}^{0}=(2 n+1) \rho_{e}^{0}=4.68 \times 10^{17} \mathrm{~kg} / \mathrm{m}^{3}(27)
$$

with $\rho_{e}^{0}=22.24 \times 10^{13} \mathrm{~kg} / \mathrm{m}^{3}$, [4].

Also, because that-according to CGT, the degenerate electrons of the protonic B-E cluster are quasi-electrons, with the charge $e^{*}=(2 / 3) \cdot e$ characteristic to quarks, by the specific dependence: $e \sim \rho_{c}(a)$, to the $\rho_{c}(r)$-density variation of the quasi-electron's magnetic moment vortex, $\Gamma_{\mu}$, it corresponds a mean radius of the $\Gamma_{\mu}$-vortex: $\eta_{\mu}$ $=0.755 \mathrm{fm},[4]$.

Supposing for $\eta_{e}, \eta_{\mu}$ an inverse proportionality with the quanta density in which is positioned the electronic centroid and with the distance between the electronic centroids, $\left(\eta_{e}^{\prime}, \eta_{\mu}^{\prime}\right) \sim 1 /\left(1 ; \rho_{c}\left(r_{c}\right)\right)$, with the previous values, we may empirically suppose the form:

$$
k_{d} \approx e^{-\frac{n \cdot \gamma}{l} \cdot r}
$$

with: $n$ - the number of bosons which forms the B-E condensate and $l$-the mean inter distance between the super dense electronic kernels (centroids).

Because that all superdense electronic centroids of the protonic B-E cluster are inside of the impenetrable quantum volume of the proton: $v_{i}\left(r_{i}\right)$ and forms the superdense kernel of protonic Compton radius, $r_{\lambda p}$, we may approximate that: $\lambda=\lambda_{k} \approx 2 r_{\lambda p} / 10 \approx 0.04 \mathrm{fm}$ and it results that:

$\gamma=\gamma_{e} \approx 5.4 \times 10^{-6}$ for the electron mass decreasing and: $\gamma=\gamma_{\mu} \approx 1.08 \times 10^{-5}$ for the electron's magnetic moment density decreasing.

The difference between $\gamma_{\mu}$ and $\gamma_{e}$ suggests the existence of a proportion $0.5>\Delta m / m_{n} \geq 0.13$ of the nucleon's mass which is in the form of vortexially retained kinetized quantons (resulted from destroied vexons).

An important consequence of the empiric expression (28) of $k_{d}$ is the conclusion that when a quasi-electron $e^{*}$ which gives the u-quark's charge inside the impenetrable quantum volume of the proton, $v_{i}\left(r_{i}\right)$, is moved in the strong interaction quantum volume $\left(r_{i}<r \leq a\right)$, by the Equation (28) and the dependence $e \sim \rho_{c}(a)$ it results that $k_{d}$ decreases to the value: $k_{d}^{\prime}=e^{-(n \gamma / l) \cdot r} \approx e^{-0.01136 \times r},(l \approx 1 \mathrm{fm} ; r$ in $[\mathrm{fm}])$, giving a value: $\eta_{\mu}^{\prime}=0.954 \mathrm{fm}$, which corresponds to: $\rho_{c}^{\prime}(a) / \rho_{c}(a)=e^{\prime} / e=0.982$, i.e. to the possibility of quark $\rightarrow$ particle transforming.

In the same time, this possibility resulted by Equation (28) explains the difficulty to find quarks in the free state, according to the theory.

For separate fermions, $\left(d_{1-2} \geq r_{e}\right), k_{d} \approx 1$, according also to the superposition principle.

\section{Conclusions}

By the previous theoretical explanations of the wave-corpuscle dualism of the photon, based to an etheronoquantonic theory of fields considering a vortexial quantum nature of the magnetic field, the revised vortexial model of pseudo-scalar photon derived from the Munera's model and the Hunter-Wadlinger model explains the ondulatory phenomena of photons by the spinorial, vortexial mass, $m_{s}$ of the component vectorial photons 
coupled with antiparallel magnetic moments, the corpuscular properties of the photons being explained by an inertial mass of the vectorial photons, $m_{w}$, equal with the spinorial mass. The model allows phenomenological microphysical explanations for the photon's wave-length variation with the value of the refraction index of the optical body and the Kerr and Cotton-Mouton effects. It is explained also microphysically the magnetic rotating polarization (the Faraday effect). The model indicates the variation of the quantum vacuum refraction index in the presence of a strong electric $E$-field or magnetic $B$-field.

The conclusion regarding the equality between the inertial and the spinorial mass of the vectorial photon and the conclusion that its spinorial mass does not contribute to its inertial mass is based to the next assumptions:

The inertial mass is given by chiral sub-solitons of high density, ("vectons" in CGT) formed and confined by the quantonic vortex $\Gamma_{\mu}=\Gamma_{A}+\Gamma_{H}$ and not vanish if the $\Gamma_{\mu}$ vortex is not vanished (i.e. the photon has also inertial rest-mass);

A quantum contributes to the inertial mass of a particle only if it is linked by a force which transmits to the particle at least partially each exterior action over it.

For a fermion, particularly for an electron, because it may be assimilated with a semi-photon, the model may explain the wave properties given by the associated de Broglie wave, also for other particles, but we observe that the proposed model corresponds to a photon for which the phase speed is equal with the group speed. By the equality: $h v=m c^{2}$, the correct form of wave-length $\lambda$ is the form: $\lambda=v_{p} / v=v_{p} \cdot h / m c^{2}=h / n \cdot m c=\lambda_{0} / n$, while the de Broglie relation gives: $\lambda_{B}=h / m v_{p}=h \cdot n / m c=n \lambda_{B}^{0}$ and for $n \neq 1$, it does not correspond to the proposed pre-quantum model of photon.

Also, the possibility of double multi-photon forming as chains of antiparallely coupled vectorial photons, may explain the double slit experiment by the conclusion that the $\Gamma_{\mu}$-vortex of the atoms which forms the slits' margins attracts the multiphoton rows with spatially variable force, determining the light's diffraction and suggests that a part of the incoherent light's energy may be emitted in the form of pairs of multiphotons coupled in antiphase, having a non-ondulatory effect of scalar wave.

\section{References}

[1] Munera, H.A. (2002) A Semiclassical Model of the Photon Based on Objective Reality and Containing Longitudinal Field Components. In: Evans, M.W., Ed., Modern Nonlinear Optics, John Wiley \& Sons Inc., Hoboken, 335-385.

[2] Lehnert, B., et al. (2000) An Extended Electromagnetic Theory. Apeiron, 7, 53.

[3] Hunter, G. and Wadlinger, R.L.P. (1989) Photons and Neutrinos as Electromagnetic Solitons. Physics Essays, 2, 158172. http://dx.doi.org/10.4006/1.3035861

[4] Arghirescu, M. (2015) A Quasi-Unitary Pre-Quantum Theory of Particles and Fields and Some Theoretical Implications. International Journal of High Energy Physics, 2, 80-103.

[5] Kiehn, R.M. (1987) The Falaco Effect. A Topological Soliton. Talk at 1987 Dynamics Days, Austin.

[6] Hunter, G., Wadlinger, L.P., et al. (2002) Gravitation and Cosmology: From the Hubble Radius to the Planck Scale. Fundamental Theories of Physics, 126, 157-166.

[7] Weinberger, P. (2008) John Kerrr and His Effect Found in 1877 and 1878. Philosophical Magazine Letters, 88, 897907. http://dx.doi.org/10.1080/09500830802526604

[8] Cotton, A. and Mouton, H. (1905) Comptes Rendus Hebdomadaires des Séances de l'Académie des Sciences Paris, 141, 317-349.

[9] Heinze, G., Hubrich, C. and Halfmann, T. (2013) Stopped Light and Image Storage by Electromagnetically Induced Transparency up to the Regime of One Minute. Physical Review Letters, 111, Article ID: 038103. http://dx.doi.org/10.1103/physrevlett.111.033601

[10] Pohl, R., Antognini, A., Nez, F., Amaro, F.D., et al. (2010) The Size of the Proton. Nature, 466, 213-216. http://dx.doi.org/10.1038/nature09250 